\title{
PSMA PET in Prostate Cancer
}

\author{
Hossein Jadvar \\ Department of Radiology, Keck School of Medicine, University of Southern California, Los Angeles, California
}

$\mathbf{M}$ olecular imaging with PET using an increasing list of biologically relevant radiotracers is paving the way for precision and personalized medicine in prostate cancer (1). Prostate-specific membrane antigen (PSMA) has received a resurgence of attention as a useful biomarker in the imaging evaluation of prostate cancer with PET. PSMA is a type II, 750-amino-acid integral membrane glycoprotein (100-120 kDa), with a 19-amino-acid intracellular component, a 24-amino-acid intramembrane segment, and a large 707-amino-acid extracellular domain (2). It has several enzymatic functions and is known to be upregulated in castrate-resistant and metastatic prostate cancer (3). Despite what the name suggests, PSMA is not specific to the prostate gland and is expressed in other normal (e.g., salivary glands, duodenal mucosa, subset of proximal renal tubular cells, and subpopulation of neuroendocrine cells in the colonic crypts) and neoplastic (e.g., subtypes of transitional cell carcinoma, renal cell carcinoma, colon carcinoma, and peritumoral and endotumoral endothelial cell of neovasculature) tissues (4). There is no known natural ligand for PSMA, and the reasons for its upregulation in prostate cancer remain unclear. PSMA undergoes constitutive internalization and as such can serve not only as an imaging biomarker but also for targeted therapy-in other words, PSMA may be useful as a target for theranostic agents (5).

The commercially available ${ }^{111}$ In-capromab pendetide targets the internal epitope of PSMA with radiolabeled mouse monoclonal antibody 7E11. However, this agent is accessible only to its target in dying or dead cells and has been technically demanding to perform, with only moderate diagnostic performance in men at high risk for lymph node or soft-tissue metastases and negative bone scintigraphy. The recently developed PET radiotracers primarily target the extracellular moiety of the PSMA (hence detecting viable cells) and include ${ }^{11} \mathrm{C}$ - (half-life, $20.3 \mathrm{~min}$ ), ${ }^{18} \mathrm{~F}$ - (half-life, $109.8 \mathrm{~min}$ ), ${ }^{68} \mathrm{Ga}$ - (half-life, $67.7 \mathrm{~min}$ ), ${ }^{89} \mathrm{Zr}$ - (half-life, $78.4 \mathrm{~h}$ ), ${ }^{64} \mathrm{Cu}$ - (half-life, $12.7 \mathrm{~h}$ ), and ${ }^{86} \mathrm{Y}$ - (half-life, $14.7 \mathrm{~h}$ ) labeled agents that involve antibodies, antibody fragments, aptamers, and PSMA inhibitors (2). In particular, many publications are now appearing in literature on the potential diagnostic utility of the Glu-NH-CO-NHLys-(Ahx)-[ $\left[{ }^{68} \mathrm{Ga}-\mathrm{HBEDD}-\mathrm{CC}\right]$ conjugate (abbreviated as ${ }^{68} \mathrm{Ga}-$ PSMA, which binds the motif glutamate-urea-lysine with the chelator HBED-CC) in prostate cancer. The normal biodistribution of this tracer shows intense uptake in salivary glands and kidneys and moderate uptake in lacrimal glands, liver, spleen,

\footnotetext{
Received Apr. 29, 2015; revision accepted Apr. 30, 2015

For correspondence or reprints contact: Hossein Jadvar, University of Southern California, 2250 Alcazar St., CSC 102, Los Angeles, CA 91107

E-mail: jadvar@med.usc.edu

Published online May 14, 2015.

COPYRIGHT (c) 2015 by the Society of Nuclear Medicine and Molecular Imaging, Inc.

DOI: 10.2967/jnumed.115.157339
}

and intestines (6). False-negative results may include prostate tumors with neuroendocrine differentiation, small tumors, and tumors located next to areas with high physiologic tracer uptake (7). False-positive results have been reported with physiologic uptake in the celiac ganglia (8).

In the primary tumor setting, a single clinical case report using a hybrid PET/MR imaging system showed high tracer localization, T2 hypointensity, and restricted diffusion at the tumor site (9). The small foci of benign prostate hyperplasia demonstrated diffuse diffusion restriction but no abnormal tracer accumulation. Although additional experience will be needed in the primary tumor setting, ${ }^{68} \mathrm{Ga}$-PSMA PET/MR imaging may provide sufficient imaging information to guide biopsy and delivery of focal therapy to the dominant aggressive lesion.

In the May 2015 issue of The Journal of Nuclear Medicine, Eiber et al. from Germany reported on a retrospective analysis of ${ }^{68} \mathrm{Ga}$-PSMA PET/CT in 248 patients with biochemical recurrence (serum PSA range, 0.2-59.4 ng/mL; median, $1.99 \mathrm{ng} / \mathrm{mL}$ ) of prostate cancer after radical prostatectomy (10). One board-certified nuclear medicine physician and 1 board-certified radiologist interpreted PET and CT images, respectively, and separately, with a final consensus interpretation. The standard of reference for the PET imaging findings was as usual mostly based on clinical or other imaging follow-up. The authors reported lesion detection rates that increased with increasing serum PSA level. The lesion detection rate for the range of key clinical interest in the low serum PSA range $(0.2-0.5 \mathrm{ng} / \mathrm{mL})$ was $58 \%$. A higher detection rate was also noted with higher PSA velocity but not with shorter PSA doubling time. The authors also found a higher lesion detection rate in patients with higher primary tumor Gleason score, but no statistically significant association was noted with regards to the use of androgen-deprivation therapy. One key question that remains unanswered by this report is the unique contribution of the nonstandard ${ }^{68} \mathrm{Ga}$-PSMA PET/CT over conventional imaging in the setting of biochemical recurrence after radical prostatectomy. Although this was tangentially addressed in this report (33\% of lesions were detected only on PET), a prospective study with well-defined inclusion criteria involving only patients with PSA relapse and no conventional imaging evidence of locally recurrent and metastatic disease would clarify the distinctive role of ${ }^{68} \mathrm{Ga}$-PSMA in this important clinical setting that then can be compared with the reported results for other relevant radiotracers. Such study design will help to elucidate the competitive advantage of ${ }^{68} \mathrm{Ga}-$ PSMA in biochemical recurrence of prostate cancer. In another study from the same group of investigators, it has been noted that ${ }^{68} \mathrm{Ga}$-PSMA may have an advantage over ${ }^{18} \mathrm{~F}$-fluorocholine (11). However, additional comparative studies will be needed to ratify this early observation and to establish the clinical relevance of potentially detecting more lesions with ${ }^{68} \mathrm{Ga}$-PSMA PET/CT in comparison to radiolabeled choline PET/CT. 
The other finding in this study was that there was no statistically significant association between the lesion detection rate and androgen-deprivation treatment. This finding is probably related to statistical power issues because it is contrary to prior reports that have demonstrated higher PSMA expression in tumor cells exposed to antihormonal treatment $(12,13)$. Future prospective studies will be needed to examine how ${ }^{68} \mathrm{Ga}$-PSMA behaves in the treatment response assessment setting with various therapy regimens (14). This will likely depend on the extent that PSMA expression may be affected by different therapies aside from cell death (which in turn can also be imaged with those tracers that target the internal epitope of PSMA). In summary, ${ }^{68} \mathrm{Ga}$-PSMA is a noteworthy radiotracer with high potential for diagnostic utility in prostate cancer. Moreover, appropriate radionuclide substitutions may provide opportunity for targeted radioisotope therapy (15). Additional prospective clinical studies in well-defined patient cohorts, clinical settings, and expected outcomes will be needed to confidently establish the role of ${ }^{68} \mathrm{Ga}$-PSMA as a major contender radiotracer in the imaging evaluation of prostate cancer with PET.

\section{DISCLOSURE}

This study was supported by National Institutes of Health, National Cancer Institute, grants R01-CA111613, R21-142426, and P30-CA014089 and the Whittier Foundation. No other potential conflict of interest relevant to this article was reported.

\section{REFERENCES}

1. Jadvar H. Molecular imaging of prostate cancer with PET. J Nucl Med. 2013;54:1685-1688.

2. Mease RC, Foss CA, Pomper MG. PET imaging in prostate cancer: focus on prostate-specific membrane antigen. Curr Top Med Chem. 2013;13:951-962.
3. Demirkol MO, Acar O, Ucar B, et al. Prostate specific membrane antigen-based imaging in prostate cancer: impact on clinical decision making process. Prostate. 2015;75:748-757.

4. Silver DA, Pellicer I, Fair WR, et al. Prostate-specific membrane antigen expression in normal and malignant human tissues. Clin Cancer Res. 1997;3:81-85.

5. Bouchelouche K, Choyke PL, Capala J. Prostate specific membrane antigen: a target for imaging and therapy with radionuclides. Discov Med. 2010;9:55-61.

6. Afshar-Oromieh A, Malcher A, Eder M, et al. PET imaging with a $\left[{ }^{68} \mathrm{Ga}\right]$ galliumlabeled PSMA ligand for the diagnosis of prostate cancer: biodistribution in humans and first evaluation of tumor lesions. Eur J Nucl Med Mol Imaging. 2013;40:486-495.

7. Chakraborty PS, Tripathi M, Agarwal KK, et al. Metastatic poorly differentiated prostatic carcinoma with neuroendocrine differentiation: negative on ${ }^{68} \mathrm{Ga}-$ PSMA PET/CT. Clin Nucl Med. 2015;40:e163-e166.

8. Krohn T, Verburg FA, Pufe T, et al. $\left[{ }^{68} \mathrm{Ga}\right]$ PSMA-HBED uptake mimicking lymph node metastasis in coeliac ganglia: an important pitfall in clinical practice. Eur J Nucl Med Mol Imaging. 2015;42:210-214.

9. Eiber M, Nekolla SG, Maurer T, Weirich G, Wester HJ, Schwaiger M. ${ }^{68} \mathrm{Ga}$ PSMA PET/MR with multimodality image analysis for primary prostate cancer. Abdom Imaging. November 21, 2014 [Epub ahead of print].

10. Eiber M, Maurer T, Souvatzoglou M, et al. Evaluation of hybrid ${ }^{68}$ Ga-PSMA ligand PET/CT in 248 patients with biochemical recurrence after radical prostatectomy. J Nucl Med. 2015;56:668-674.

11. Afshar-Oromieh A, Zechmann CM, Malcher A, et al. Comparison of PET imaging with a ${ }^{68} \mathrm{Ga}$-labeled PSMA ligand and ${ }^{18} \mathrm{~F}$-choline based PET/CT for the diagnosis of recurrent prostate cancer. Eur J Nucl Med Mol Imaging. 2014; 41:11-20.

12. Wright GL Jr, Grob BM, Haley C, et al. Upregulation of prostate-specific membrane antigen after androgen-deprivation therapy. Urology. 1996;48: 326-334.

13. Afshar-Oromieh A, Avtzi E, Giesel FL, et al. The diagnostic value of PET/CT imaging with the ${ }^{68} \mathrm{Ga}$-labeled PSMA ligand HBED-CC in the diagnosis of recurrent prostate cancer. Eur J Nucl Med Mol Imaging. 2015;42:197-209.

14. Uprimny C, Kroiss A, Nilica B, et al. ${ }^{68} \mathrm{Ga}$-PSMA ligand PET versus $\left({ }^{18} \mathrm{~F}\right) \mathrm{F}-\mathrm{NaF}$ PET: evaluation of response to ${ }^{223} \mathrm{Ra}$ therapy in a prostate cancer patient. Eur $J$ Nucl Med Mol Imaging. 2015;42:362-363.

15. Baum RP, Kulkarni H, Weineisen M, et al. Advancing personalized nuclear medicine by theranostics of prostate cancer using Ga-68 and Lu-177 labeled PSMA small molecules. J Nucl Med. 2015;56(suppl. 1):22. 